Artigo Original

\title{
Uso de visão periférica na interceptação de alvos móveis
}

\author{
Leandro Quedas Caminha \\ Raymundo Machado AZEVEDO Neto \\ Luis Augusto Teixeira
Laboratório Sistemas Motores Humanos, Escola de Educação Física e Esporte, Universidade de São Paulo, São Paulo, SP, Brasil

\begin{abstract}
Resumo: O uso de visão periférica para organização e reorganização de uma ação interceptativa foi investigada em adultos jovens. Erros temporais e variáveis cinemáticas foram avaliados na interceptação de um alvo móvel virtual, em situações em que sua velocidade inicial era mantida inalterada ou era inesperadamente reduzida. A observação da aproximação do alvo foi feita por meio de perseguição contínua (visão focal) ou mantendo-se o foco visual fixo na origem da trajetória ou ponto de contato (visão periférica). Os resultados mostraram que o foco visual no ponto de contato levou a erros temporais semelhantes à visão focal, porém com perfis cinemáticos distintos, enquanto que o foco visual na origem levou a um desempenho empobrecido.
\end{abstract}

Palavras-chave: Percepção Visual. Movimento. Neuropsicologia.

\section{Use of peripheral vision in the interception of moving targets}

\begin{abstract}
Use of peripheral vision to organize and reorganize an interceptive action was investigated in young adults. Temporal errors and kinematic variables were evaluated in the interception of a virtual moving target, in situations in which its initial velocity was kept unchanged or was unexpectedly decreased. Observation of target approach was made through continuous visual pursuit (focal vision) or keeping visual focus at the origin of the trajectory or at the contact spot (peripheral vision). Results showed that visual focus at the contact spot led to temporal errors similar to focal vision, although showing a distinct kinematic profile, while focus at the origin led to an impoverished performance
\end{abstract}

Key Words: Perception. Movement. Neuropsychology.

\section{Introdução}

A habilidade de realizar ações de interceptação de alvos móveis tem sido amplamente utilizada no estudo da integração entre informação visual e organização motora, por causa das restrições particulares impostas ao sistema visuomotor (WILLIAMS et al., 1999). Uma característica particular nestas ações consiste no fato de que uma ação interceptativa bem sucedida requer a extração de informação visual sobre a aproximação do alvo móvel, permitindo estimar o tempo para contato entre o alvo e o interceptador. A extração de informação visual tem sido majoritariamente estudada em função do uso de variáveis óticas associadas à visão focal, tais como a taxa de dilatação da imagem do alvo na retina (LEE et al., 1983; SAVELSBERGH et al., 1991; MICHAELS et al., 2001) ou da constrição do ângulo formado pelos eixos olho-alvo e olhomão (LEE et al., 2001; CALJOUW et al., 2004), conforme o alvo se aproxima do interceptador. No entanto, a extração de informação visual sobre um alvo em movimento poderia ser feita a partir de uma variedade de estratégias visuais, empregando também visão periférica.

Diferentes estratégias de visualização de um alvo móvel implicam diferentes formas de estimativa do tempo para contato. Para tal finalidade, são concebidos dois sistemas perceptomotores: imagem-retina e olhos-cabeça. O sistema imagem-retina consiste em estabilizar a cabeça e os olhos em uma posição fixa e deixar que a imagem do alvo transite pela retina. Neste caso, o olhar poderia ser fixado na posição de origem do deslocamento do alvo, fazendo com que a imagem transitasse da fóvea para a periferia do campo visual. Alternativamente, a fixação visual poderia ser feita no local estimado de contato, fazendo com que a imagem do alvo transitasse no sentido contrário, do campo visual periférico para a fóvea. No sistema olhos-cabeça o interceptador procura fovear a imagem durante todo o seu percurso. Para tanto, gera movimentos da cabeça em coordenação com movimentos dos olhos, fazendo com que a imagem permaneça projetada a maior parte do tempo na região visual com maior resolução espacial. Estas são 
estratégias visuais qualitativamente distintas, porém em situações naturais de rastreamento de alvos visuais elas são usadas em combinação (BAHILL; LARITZ, 1984).

O processamento da informação visual gerada pela incidência de luz sobre a retina é distinto entre as regiões focal e periférica, desde a distribuição de cones e bastonetes ao longo dessas áreas até o núcleo geniculado lateral, seguindo caminhos neurais distintos através das vias parvo e magno celulares. Do córtex visual primário às áreas de associação sensoriais e motoras, as aferências visuais provenientes da fóvea e periferia da retina são conduzidas pelos caminhos dorsal e ventral, que têm sido considerados como vias de processamento de informação visual para ação e percepção, respectivamente (MILNER; GOODALE, 2008). Enquanto a via dorsal é principalmente composta por informação visual proveniente da periferia da retina (via magno-celular), o fluxo ventral é composto em sua maioria por informação visual proveniente da fóvea (via parvo-celular) (WURTZ; KANDEL, 2000). A partir deste quadro anatômico e funcional do sistema visual, é concebível que a pobre acuidade visual proveniente da visão periférica não esteja necessariamente vinculada a uma fraca capacidade de guiar ações motoras. Tem sido demonstrada, por exemplo, a capacidade de humanos realizarem ajustes finos no movimento de preensão de blocos de madeira ao empregar a visão periférica, embora os participantes tenham sido incapazes de relatar com precisão o comprimento do objeto (MURPHY; GOODALE, 1994; BROWN et al., 2005). Além disso, tem sido demonstrado que a disponibilidade de visão periférica da mão ao executar tarefa de alcançar um alvo fixo aumenta a precisão direcional do movimento (ABAHNINI; PROTEAU, 1999; PROTEAU et al., 2000). Estes resultados indicam a relevância da visão periférica em orientar ações dirigidas a alvos estáticos. No entanto, pouca informação tem sido gerada sobre - papel da visão periférica em situações dinâmicas, nas quais as propriedades temporais de um alvo móvel devem ser estimadas para realizar a ação interceptativa.

Algum insight sobre a funcionalidade da visão periférica em situações dinâmicas pode ser obtido de estudos enfocando estratégias visuais em tarefas com alvos móveis em situações naturais. Nestes trabalhos tem sido observado que os participantes geralmente optam por manter a imagem do objeto sobre a fóvea em momentos críticos da preparação da ação (LAND; MCLEOD, 2000; RODRIGUES et al., 2002). Por outro lado, foi demonstrado que a observação em tarefa de rebatida por indivíduos habilidosos não ocorre de forma contínua, havendo trechos do deslocamento em que a imagem do alvo não é projetada sobre a fóvea (LAND; MCLEOD, 2000). Entretanto, como nestes estudos a estratégia visual foi variada livremente pelos executantes, torna-se impraticável estimar a funcionalidade da visão periférica na interceptação de alvos em movimento. Uma exceção a esta limitação é encontrada em um estudo em que bolas de tênis de mesa eram lançadas em diferentes velocidades para o agarrador, o qual usava apenas visão focal ou apenas visão periférica para rastrear a aproximação da bola (MONTAGNE et al., 1993). Os resultados revelaram que nas condições de maior exposição visual (períodos de deslocamento da bola de 330 e $370 \mathrm{~ms}$ ) não houve diferença entre as condições visuais. A condição de menor exposição visual (260 ms), entretanto, revelou que a visão periférica levou a uma proporção maior de bolas agarradas em relação à perseguição visual contínua. Este achado sugere que a visão periférica pode ser efetiva em fornecer informação sobre o tempo para contato com o alvo móvel. A mensuração do desempenho na tarefa de preensão empregada por Montagne, entretanto, não discriminou os componentes espacial e temporal do desempenho. Essa limitação da avaliação dos resultados, assim, impede uma conclusão mais segura sobre o papel da visão periférica em prover informação sobre o tempo para contato com um alvo móvel em tarefas interceptativas.

No presente estudo, a capacidade de utilizar a visão periférica para regulação de uma ação interceptativa foi avaliada por meio do paradigma de modificação probabilística da velocidade de um alvo móvel (TEIXEIRA et al., 2005; TEIXEIRA;CHUA et al., 2006a; b; TEIXEIRA; FRANZONI et al., 2006). A estratégia experimental consistiu em reduzir inesperadamente a velocidade de um alvo móvel em diferentes porções do campo visual, comparando estratégias visuais baseadas em visão focal ou visão periférica. Dessa forma, foi avaliada não apenas a capacidade de extrair informação visual útil à interceptação em situações de velocidade constante, mas também 
a capacidade de promover a reorganização da ação interceptativa em tempo real em resposta à modificação do tempo para contato imposta pela mudança de velocidade do alvo.

\section{Método}

\section{Participantes}

Participaram do experimento 12 estudantes universitários de ambos os sexos, com idade média de 20,3 anos ( $D P=1,6)$ e estatura média de $171 \mathrm{~cm}(\mathrm{DP}=11,5)$. Todos possuíam visão normal ou corrigida para a normal e eram novatos na tarefa experimental. Não foram aceitos participantes que usassem óculos, pois sua armação poderia restringir a visão periférica. Os participantes assinaram um termo de consentimento livre e esclarecido para ingressar no estudo. Os procedimentos experimentais foram aprovados por Comitê de Ética local.

\section{Equipamento e Tarefa}

O equipamento consistiu em um trilho eletrônico para respostas antecipatórias, constituído por uma estrutura metálica de 1,33 m de comprimento, $8 \mathrm{~cm}$ de largura e $6 \mathrm{~cm}$ de altura. Essa estrutura sustenta um feixe de diodos emissores de luz, cor vermelha, de $1 \mathrm{~cm}$ de diâmetro, alinhados em linha reta sem espaçamento entre diodos adjacentes. Os diodos eram acesos e apagados em sequência, produzindo uma nítida percepção de movimento contínuo de um ponto de luz (doravante denominado alvo) em velocidade constante. As especificações do deslocamento do alvo eram controladas por software. Em uma das pontas do trilho havia um transdutor de força embutido em uma hemibola de tênis preenchida com massa plástica. Esse transdutor é conectado a um microcomputador, que registrava a diferença de tempo entre o toque no transdutor e o instante de acendimento do último diodo da sequência com precisão de $1 \mathrm{~ms}$. $O$ trilho ficava a $70 \mathrm{~cm}$ de altura do solo e os participantes permaneciam em pé ao lado de sua extremidade. $O$ alvo luminoso percorria o trilho da extremidade distal até a extremidade proximal ao participante. A tarefa experimental consistia em contatar a hemibola ao final do trilho com uma raquete de badminton, utilizando um movimento semelhante a uma batida do tipo forehand do jogo de tênis. $O$ objetivo nesta tarefa era contatar a hemibola simultaneamente com o momento em que o alvo chegava ao último diodo do trilho. A amplitude de movimento foi delimitada. Para tanto, os participantes eram solicitados a iniciar seus movimentos com a raquete encostada em um anteparo vertical distando $60 \mathrm{~cm}$ da hemibola (Figura 1)

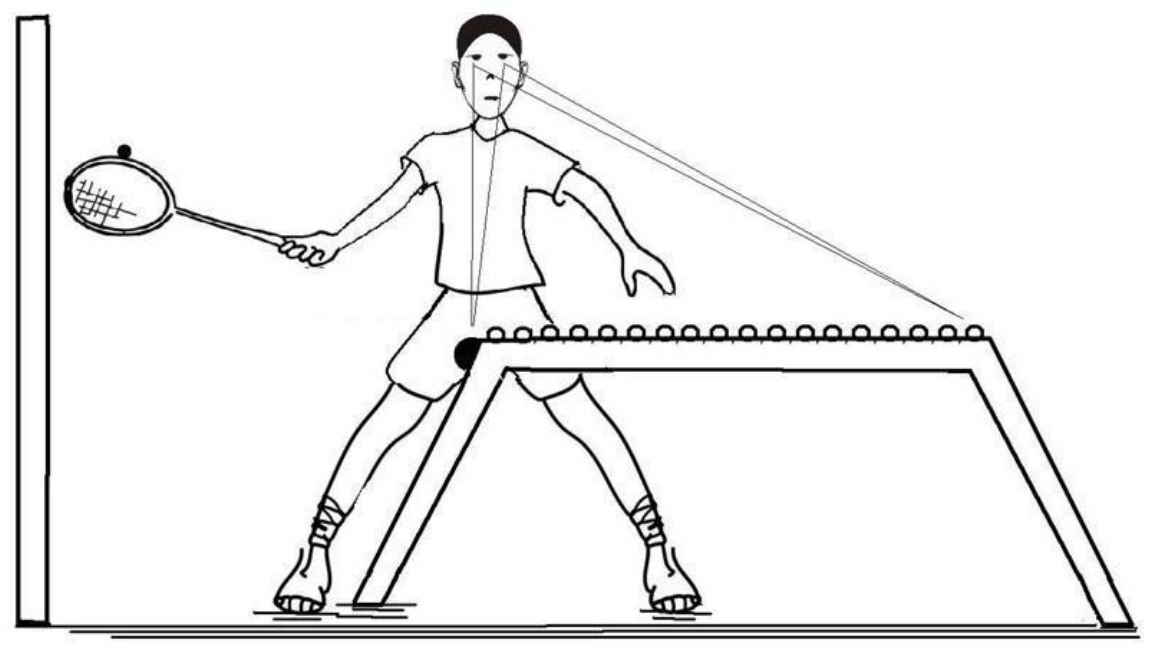

Figura 1. Representação da Situação de Foco no Contato, Mostrando a Ação Interceptativa e o Trilho Eletrônico para Respostas Antecipatórias.

Todas as tentativas foram efetuadas com 0 braço direito. A posição do indivíduo em relação ao trilho foi estipulada de forma que, no plano frontal, a pessoa estivesse paralela ao trilho e, no plano sagital, o centro do corpo estivesse na direção da hemibola. $O$ afastamento do trilho foi estipulado em $50 \mathrm{~cm}$, medidos a partir da extremidade frontal do pé. Durante todo o experimento, o ambiente permaneceu com a iluminação reduzida para destacar 0 alvo luminoso, mas sem prejudicar a visão do participante de seu braço de execução. 
Para registro dos movimentos da raquete, foi utilizada uma câmera optoeletrônica (Qualisys). Os movimentos da raquete foram registrados pelo rastreamento de um marcador refletivo disposto na parte superior da cabeça da raquete, no mesmo eixo vertical da região que fazia contato com a hemibola. Os dados do marcador foram adquiridos com frequência de $240 \mathrm{~Hz}$.

\section{Delineamento Experimental e Procedimentos}

Para exigir correções do movimento, o alvo foi configurado de maneira que poderia se deslocar em velocidade constante ou apresentar redução instantânea de velocidade ao longo de seu curso. A probabilidade de ocorrência de redução de velocidade foi estabelecida em $40 \%$. Quando o tempo de deslocamento do alvo permanecia constante, sua duração era de $667 \mathrm{~ms}(2 \mathrm{~m} / \mathrm{s})$. $\mathrm{Em}$ relação ao tempo para redução de velocidade, foram introduzidas duas condições. Estas foram planejadas com o propósito de que a demanda de correção ocorresse em momentos distintos da ação. A redução probabilística de velocidade do alvo ocorria em dois instantes: 100 ms (R100) ou 250 ms (R250) após o início de seu deslocamento pelo trilho eletrônico. Com a redução de velocidade do alvo era induzido um erro temporal, conforme apresentado na Tabela 1. Caso o participante não promovesse correções temporais em seus movimentos nas tentativas com redução de velocidade, portanto, a ação seria concluída antes do tempo adequado para uma interceptação precisa. As reduções de velocidade ocorriam a 1,13 e $0,83 \mathrm{~m}$ de distância em relação à extremidade proximal do trilho, respectivamente para R100 e R250.

Tabela 1. Parâmetros Temporais Envolvidos nas Situações R100 e R250 (ms)

\begin{tabular}{lccc}
\hline & R100 & R250 \\
\hline Tempo até a redução de & 100 & 250 \\
velocidade & & 567 & 417 \\
Tempo restante em vel. constante & 800 & 715 \\
Tempo restante após redução & 233 & 298 \\
Erro temporal induzido & 900 & 965 \\
\hline Tempo total com redução & & \\
\hline
\end{tabular}

A tarefa de interceptação foi desempenhada sob três condições visuais: (a) manter o foco visual sobre 0 alvo durante todo 0 seu deslocamento, por meio de movimentos da cabeça e dos olhos (perseguição contínua, PC); (b) manter o foco visual no primeiro diodo distal do trilho durante todo o deslocamento do alvo, de forma que a projeção da imagem na retina se deslocasse da fóvea para a periferia do campo visual (foco na origem, FO); (c) manter o foco visual no primeiro diodo proximal do trilho, de forma que a projeção da imagem na retina se deslocasse da periferia do campo visual para a fóvea (foco no contato, FC).

As duas condições de tempo para redução de velocidade foram aplicadas em diferentes blocos experimentais. Assim, os participantes foram submetidos a seis blocos de tentativas, resultantes da combinação de dois fatores: foco visual (3) e tempo de redução de velocidade (2). $\mathrm{Na}$ condição $\mathrm{FO}$, o alvo poderia ter sua velocidade reduzida em uma excentricidade ocular de $5^{\circ}$ (R100) ou 15을 (R250). Na condição FC, o alvo poderia ter sua velocidade reduzida em uma excentricidade de 45 (R100) ou 35 (R250). O ângulo visual para observação de todo 0 deslocamento do alvo nas condições de foco visual fixo foi de $50^{\circ}$ (os valores angulares são aproximados, sofrendo pequena variação devido às diferentes estaturas entre os participantes). A cabeça dos participantes não foi fixada por qualquer dispositivo mecânico, mas nas situações de foco visual fixo eles eram instruídos a manter a posição da cabeça imóvel. Durante a sessão experimental, havia um experimentador especialmente dedicado a monitorar se as instruções de posição da cabeça e foco visual estavam sendo seguidas pelos participantes.

A estabilização do desempenho foi realizada através da prática de 240 tentativas, sendo 40 para cada um dos 6 blocos experimentais, com conhecimento de resultados sobre 0 erro constante temporal. Dois minutos após a fase de estabilização eram realizadas as tentativas probatórias. Antes do início de cada bloco de tentativas probatórias, os participantes realizavam 20 tentativas de estabilização com conhecimento de resultados. Na sequência, as condições experimentais eram avaliadas em blocos de 30 tentativas, nas quais não era oferecido conhecimento de resultados. Foi adotado um intervalo de 2 min. entre a execução dos blocos experimentais. A ordem dos blocos foi contrabalançada entre os participantes. 


\section{Análise dos Dados}

Foram selecionadas as 10 primeiras tentativas sem falhas de registro (erro temporal $e$ cinemática) em velocidade constante e em velocidade alterada para análise, descartando-se as demais. As variáveis dependentes foram erro temporal e variáveis cinemáticas relacionadas à organização temporal da ação. O erro temporal foi avaliado em termos de direção (erro constante, movimentos adiantados $=$ sinal negativo, atrasados $=$ sinal positivo), magnitude (erro absoluto, valores de erro constante em módulo) e variabilidade intraindividual (erro variável, desvio padrão de erro constante). As variáveis cinemáticas avaliadas foram o tempo de iniciação, correspondendo ao tempo entre os instantes de início do deslocamento do alvo e de início da ação; tempo para contato, correspondendo ao intervalo entre os instantes de iniciação da ação e contato com a hemibola; e tempo de movimento, correspondendo à soma do tempo de iniciação com o tempo para contato. A análise estatística foi feita por meio de análises de variância de três fatores, 3 (visão: PC $\times$ FO $\times$ FC) $\times 2$ (velocidade: constante $\times$ reduzida) $\times 2$ (tempo: R100 $\times$ R250), com medidas repetidas nos três fatores. As comparações posteriores foram realizadas por meio da prova de Newman-Keuls. O nível de significância foi estabelecido em 0,05 em todas as comparações.

\section{Erro Temporal}

A análise do erro constante indicou efeito significante para o fator principal velocidade $[F(1,11)=80,77, p<0,01]$, interação entre os fatores principais velocidade e tempo $[F(1,11)=$ $20,62, p<0,01]$ e entre os três fatores $[F(2,22)=$ $6,80, p<0,01]$. As comparações posteriores para o fator velocidade indicaram que a condição velocidade constante do alvo induziu tendência de atraso do contato, enquanto que a velocidade reduzida induziu tendência de adiantamento (Figura 2A). As comparações para a interação entre velocidade e tempo indicaram que na condição R250, comparada com a condição R100, houve maior antecipação na condição velocidade reduzida, e também maior atraso na condição velocidade constante. As análises posteriores para a interação entre os três fatores indicaram que a condição $F O$ foi a maior responsável pela diferença de erro entre $\mathrm{R} 100 \mathrm{e}$ R250. Na condição R250, os participantes apresentaram desempenho mais adiantado em FO do que nas condições PC e FC para a condição velocidade reduzida, assim como maiores atrasos em comparação às demais estratégias visuais para velocidade constante.

A análise do erro absoluto indicou efeitos significantes para os fatores principais visão $[F(2,22)=17,89, p<0,01]$ e tempo $[F(1,11)=$ $23,03, \quad p<0,01]$, assim como interação significante entre visão e velocidade $[F(2,22)=$ $4,90, p<0,05]$ e entre visão e tempo $[F(2,22)=$ $3,49, p<0,05]$. As comparações para $\circ$ fator principal visão mostraram que os participantes, na condição $\mathrm{FO}$, foram menos precisos do que nas condições PC e FC, não havendo diferenças entre as duas últimas condições. As comparações posteriores para o fator principal tempo indicaram maior precisão em R100 do que em R250 (Figura 2B). A decomposição da interação entre visão e velocidade indicou que a diferença na precisão encontrada entre R100 e R250 ocorreu apenas nas condições PC e FO. As comparações para a interação entre os fatores visão e tempo indicaram que quando os participantes foram submetidos à condição $\mathrm{FO}, \mathrm{a}$ velocidade constante do alvo induziu erro significantemente inferior do que a velocidade reduzida do alvo, efeito que não ocorreu para PC e FC. Nestas últimas condições, a precisão temporal foi semelhante entre as condições velocidade constante e reduzida do alvo.

A análise do erro variável indicou efeitos significantes para os fatores principais visão $[F(2,22)=10,68, p<0,01]$, tempo $[F(1,11)=5,03$, $p=0,046]$ e velocidade $[F(1,11)=76,37, p<$ $0,01]$. Foi detectada também interação entre os fatores visão e velocidade $[F(2,22)=4,58, p=$ $0,02]$. As comparações indicaram que em $F O$ houve maior variabilidade de erro do que em $\mathrm{PC} e$ FC, não havendo diferença entre estas últimas condições. As análises posteriores para o fator tempo indicaram que em R250 houve maior erro variável do que em R100. Foi detectado também para o fator velocidade que a situação de velocidade reduzida induziu maior variabilidade de erro do que a situação de velocidade constante (Figura $2 \mathrm{C}$ ). A análise da interação entre os fatores visão e velocidade indicou que na condição $\mathrm{FC}$ foram observados valores maiores do que na condição PC, embora em velocidade constante não tenha sido detectada diferença entre estas condições. 

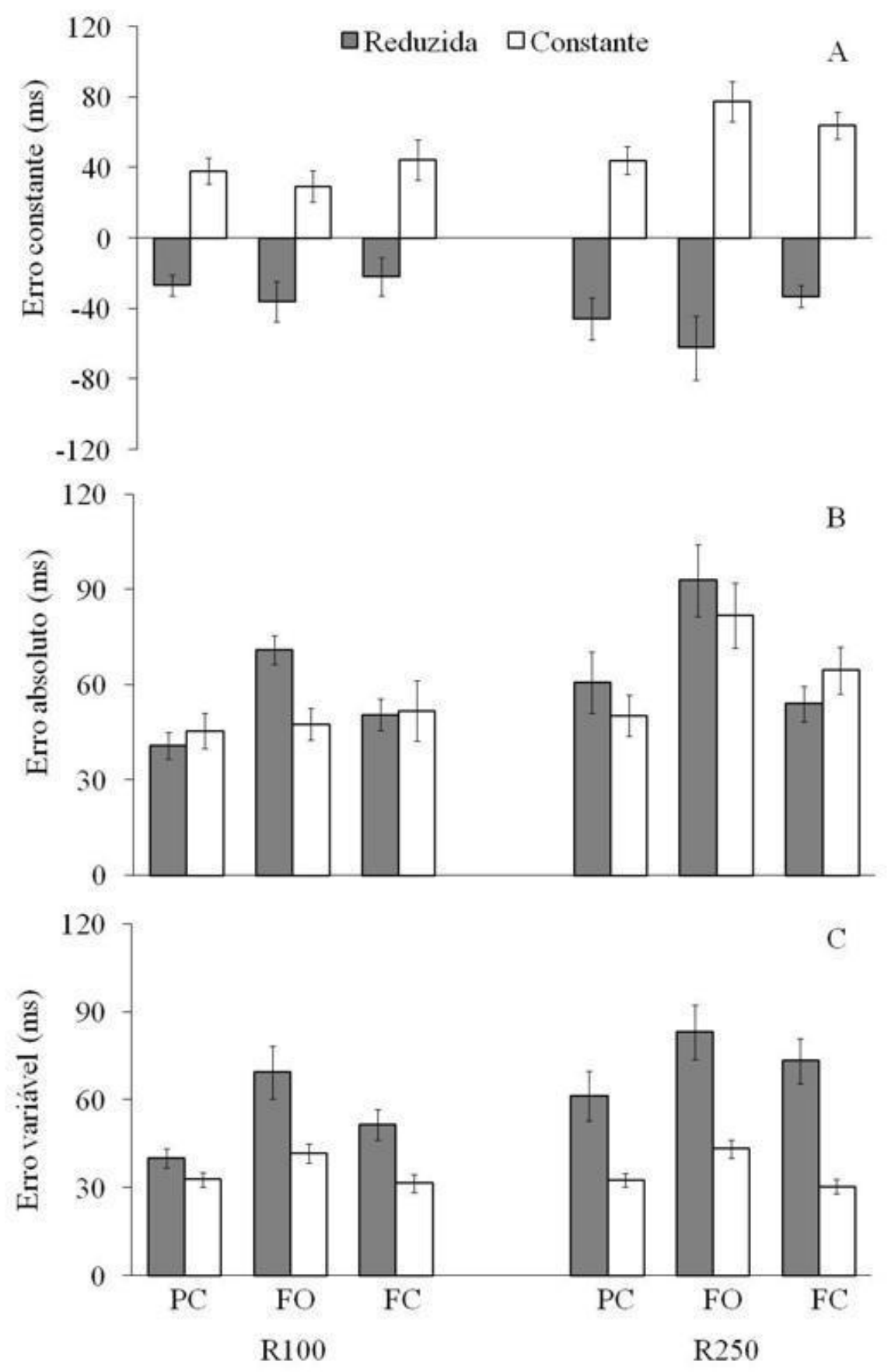

Figura 2. Valores Médios dos Erros Temporais (ms) Constante (A), Absoluto (B) e Variável (C), em Função da Condição Visual (Perseguição Contínua, PC; Foco na Origem, FO; e Foco no Contato, FC), Velocidade (Constante x Reduzida) e Tempo de Redução de Velocidade (R100 x R250); Traços Verticais Representam o Erro Padrão.

\section{Cinemetria}

A análise do tempo de iniciação identificou efeitos significantes para os fatores principais visão $[F(2,22)=15,18, p<0,01]$, tempo $[F(1,11)=$ $8,45, p=0,01]$ e velocidade $[F(1,11)=76,37, p<$ $0,01]$. As comparações apontaram que na condição FC houve tempo de iniciação significantemente superior do que nas condições $\mathrm{PC}$ e $\mathrm{FO}$, que não foram diferentes entre si. Em relação ao fator tempo, na condição $\mathrm{R} 100$ foi detectado tempo de iniciação significantemente inferior a R250. As análises posteriores para o fator velocidade indicaram tempo de iniciação superior quando a velocidade do alvo era reduzida. No entanto, as comparações entre os efeitos simples demonstraram que o efeito foi significante somente na condição R250 para $\mathrm{PC}$ e FC (Figura 3A). 

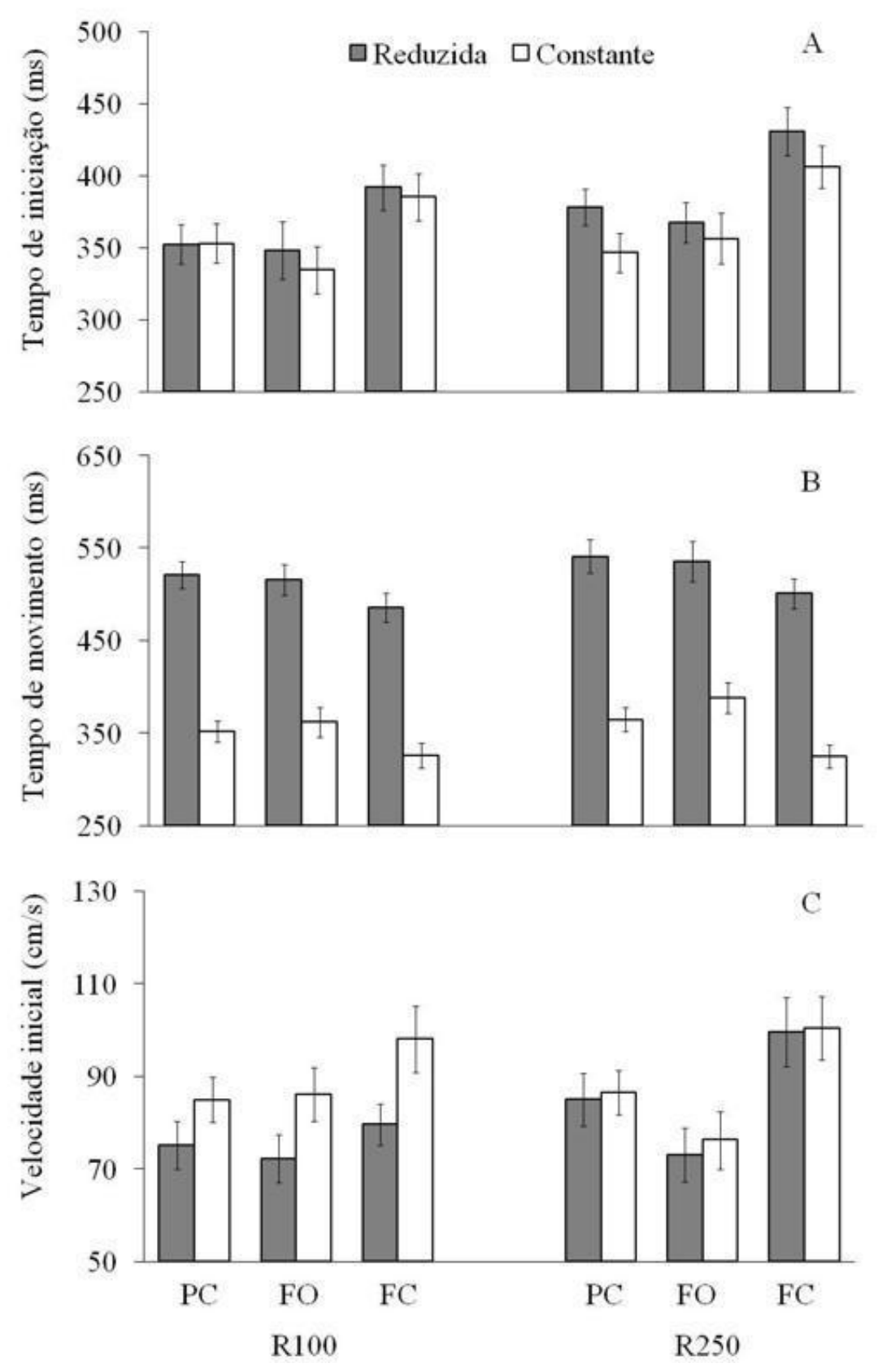

Figura 3. Valores Médios (ms) do Tempo de Iniciação (A), Tempo de Movimento (B) e Velocidade Inicial do Movimento (C), em Função da Condição Visual, Velocidade e Tempo de Redução de Velocidade; Traços Verticais Representam o Erro Padrão.

A análise do tempo de movimento indicou efeito significante para os fatores principais visão $[F(2,22)=6,70, p<0,01]$ e velocidade $[F(1,11)=$ $273,54, p<0,01]$. As comparações posteriores indicaram que o tempo de movimento na condição $\mathrm{FC}$ foi significantemente inferior à $\mathrm{PC} e$ FO, com estas duas condições não diferindo entre si. As comparações posteriores para o fator velocidade identificaram que o tempo de movimento na velocidade reduzida foi superior àquele observado sob velocidade constante (Figura 3B).

$\mathrm{Na}$ análise da velocidade inicial, foram identificados efeitos significantes para os fatores principais visão $[F(2,22)=7,62, p<0,01]$, velocidade $[F(1,11)=18,80, p<0,01]$ e para interação entre tempo e velocidade $[F(1,11)=$ $5,60, p<0,01]$. As comparações posteriores indicaram que a velocidade inicial foi significantemente superior na condição FC em 
comparação a PC e FO. A decomposição da interação entre os fatores tempo e velocidade indicou que em R100, quando o alvo deslocavase em velocidade constante, a velocidade inicial de movimento foi superior à situação com redução de velocidade do alvo. Tal diferença não foi observada em R250 (Figura 3C).

\section{Discussão}

O objetivo desta investigação foi avaliar a capacidade da visão periférica em fornecer informação útil à interceptação de alvos móveis. Para isso, foram analisadas tanto situações com velocidade constante quanto com redução inesperada da velocidade de um alvo móvel virtual. A redução de velocidade do alvo induziu maior erro variável e tendência direcional de erro temporal distinta da situação de velocidade constante, indicando que a estratégia experimental gerou efetivamente uma perturbação à execução do movimento. A maior variabilidade na situação de redução de velocidade do alvo indica a tentativa dos participantes de ajustarem o tempo de movimento da ação interceptativa ao novo tempo para contato, seguindo a redução de velocidade do alvo. No entanto, nas tentativas em que o alvo tinha sua velocidade reduzida, a tendência geral foi de adiantamento da resposta, independentemente da condição visual. Esse aspecto denota que os movimentos foram apenas parcialmente corrigidos, apesar de haver um tempo relativamente longo para promover os devidos ajustes nos movimentos. Esta dificuldade de reorganizar ações interceptativas em resposta a mudanças inesperadas de velocidade do alvo estão em conformidade com observações prévias (TEIXEIRA; CHUA et al., 2006a; b; AZEVEDO NETO; TEIXEIRA, 2009), e indicam que este é um processo de ordem superior de integração visuomotora, demandando maior tempo para implementar correções no movimento original.

Os resultados revelaram que as condições $\mathrm{PC}$ e FC levaram a erros temporais semelhantes, enquanto que em FO houve erros de maior magnitude. Esse dado revela que, nas situações avaliadas, o uso do sistema imagem-retina com deslocamento da imagem da periferia em direção à fóvea foi tão efetivo quanto o uso do sistema olhos-cabeça. Um aspecto a ser destacado nesta comparação é que na condição FC os movimentos foram iniciados tardiamente. Aparentemente, o atraso no início do movimento é devido à demora na identificação do início do deslocamento do sinal, por ocorrer em posição periférica remota do campo visual. No entanto, este atraso foi plenamente compensado durante a execução da ação, por meio de desenvolvimento de maior velocidade no início do movimento e menor tempo de duração da ação. Uma possível interpretação deste resultado é que o desempenho obtido na condição de foco visual no ponto de contato é devido à maior definição espacial da visão focal no local de interceptação do alvo. No entanto, é importante perceber que o tempo de iniciação médio foi ao redor de $400 \mathrm{~ms}$, para tempos de deslocamento do alvo entre 667 ms (velocidade constante) e $900 \mathrm{~ms}$ (R100). Consequentemente, os movimentos de interceptação foram iniciados quando a imagem do alvo ainda estava projetada em regiões periféricas da retina. Outro ponto a ser considerado a este respeito é que na condição FC a velocidade inicial do movimento foi inferior na situação de redução de velocidade em comparação à situação de velocidade constante do alvo. Este dado indica que a alteração de velocidade ocorrida em região periférica remota do campo visual foi utilizada no planejamento original da ação. Um elemento adicional a ser considerado nesta discussão é que o período de latência de 150-200 ms para uso de informação visual no controle motor (GEORGOPOULOS et al., 1981; CARNAHAN; MARTENIUK, 1994; BOCK; JUNGLING, 1999; BOULINGUEZ; NOUGIER, 1999; ENGEL; SOECHTING, 2000; SAUNDERS; KNILL, 2004; LE RUNIGO et al., 2005) limita a capacidade de usar a visão quando a imagem do alvo se encontra próxima à fóvea. Estas observações conduzem à conclusão de que a translação da imagem em regiões periféricas remotas da retina foi usada de forma efetiva para regulação dos movimentos de interceptação na condição FC.

Outro aspecto a ser considerado sobre o desempenho observado na condição FC foi que, tanto na situação de velocidade constante quanto na situação de redução de velocidade, a visão periférica foi tão efetiva quanto perseguição visual contínua em orientar movimentos temporalmente precisos. Este dado revela a sensibilidade da visão periférica em detectar mudanças de velocidade do alvo e introduzir os ajustes necessários à ação interceptativa. Sobre este ponto, cabe a observação de que a maior frequência de eventos era de velocidade 
constante do alvo, o que tem mostrado induzir o executante a pré-organizar seus movimentos para esta velocidade de deslocamento do alvo (TEIXEIRA et al., 2005; AZEVEDO NETO; TEIXEIRA, 2009). Portanto, os resultados aqui apresentados consistem em evidência original de que a visão periférica foi efetiva não apenas na organização temporal de ações interceptativas, mas também na sua reorganização em resposta a uma redução inesperada de velocidade do alvo.

Um dado de interesse nos resultados foi a similaridade de características cinemáticas entre as condições PC e FO, a despeito de a última ter levado a valores de erro temporal mais elevados. Este achado é contraditório com os resultados de Montagne et al. (1993), mostrando que em tarefa de agarrar bolas rápidas a fixação do foco visual no ponto de lançamento das bolas levou a resultados tão bons ou melhores em comparação à perseguição visual contínua de sua aproximação. No entanto, um ponto deve ser considerado nesta comparação. No estudo de Montagne as bolas viajavam em velocidades elevadas, com intervalo entre seu disparo e chegada à mão do participante variando entre 260 e 370 ms. Neste caso, o atraso visuomotor (SMEETS et al., 1998) restringe o uso de informação visual a apenas a porção inicial da trajetória da bola, enquanto que a velocidade de deslocamento do alvo visual limita a capacidade de efetiva perseguição visual (BAHILL; LARITZ, 1984). No presente estudo, por outro lado, o tempo relativamente longo entre o início da trajetória do alvo/redução de sua velocidade e o momento devido de interceptação parece limitar o uso da informação visual da parte inicial do deslocamento do alvo. Aparentemente a informação visual mais precisa originária da porção inicial da trajetória do alvo na condição visual FO necessita ser atualizada com aferência visual mais recente e precisa da variação de posição do alvo, alimentando adequadamente um modelo mental empregado para estimar o tempo para contato (cf. DUBROWSKI et al., 2000; AZEVEDO NETO; TEIXEIRA, 2009). Um tempo longo entre a aferência visual mais precisa da visão focal e o momento de interceptação poderia tornar imprecisa a estimativa de tempo para contato por meio deste processo de integração visuomotora. Este ponto de vista é reforçado pela observação de que a redução de velocidade na situação R100 (5º de ângulo visual) levou a erros absolutos maiores do que na situação de velocidade constante em FO, mas não nas demais condições visuais.

A partir dos resultados aqui apresentados, foi evidenciado o potencial da visão periférica em fornecer informação útil ao controle de ações interceptativas de alvos móveis. Estes resultados, assim, complementam observações prévias da contribuição da visão periférica do braço de execução de movimentos orientados ao contato com alvos espaciais estáticos (e.g., PROTEAU et al., 2000; BÉDARD; PROTEAU, 2003; 2004). De particular importância para a teorização da integração visuomotora em ações interceptativas foi a demonstração da efetividade de diferentes fontes de aferência visual para prover informação sobre tempo para contato. Este achado corrobora a proposição de que múltiplas fontes de informação visual são usadas na interceptação de um alvo móvel (TRESILIAN, 1999). A partir dos resultados aqui relatados, portanto, foi mostrado que os sistemas olhos-cabeça e imagem-retina poderiam ser usados de forma flexível a fim de extrair informação útil à interceptação, a despeito de a visão focal ser usualmente empregada de forma prioritária. Permanece em aberto, entretanto, a questão da extensão em que a relação aqui retratada é modulada pelo aumento da velocidade do alvo, de forma mais similar a situações de interceptação de alvos móveis reais, como ocorre em contextos esportivos.

\section{Referências}

ABAHNINI, K.; PROTEAU, L. The role of peripheral and central visual information for the directional control of manual aiming movements. Canadian Journal of Experimental Psychology, v.53, n.2, Jun, p.160-75, 1999.

AZEVEDO NETO, R. M.; TEIXEIRA, L. A. Control of interceptive actions is based on expectancy of time to target arrival. Experimental Brain

Research, v.199, n.2, Nov, p.135-43, 2009.

BAHILL, A. T.; LARITZ, T. Why can't batters keep their eyes on the ball? American Scientist, v.72, p.249-253, 1984.

BÉDARD, P.; PROTEAU, L. On the role of peripheral visual afferent information for the control of rapid video-aiming movements. Acta Psychologica (Amst), v.113, n.1, May, p.99-117, 2003.

BÉDARD, P.; PROTEAU, L. On-line vs. off-line utilization of peripheral visual afferent information to ensure spatial accuracy of goal-directed 
movements. Experimental Brain Research, v.158, n.1, Sep, p.75-85, 2004.

BOCK, O.; JUNGLING, S. Reprogramming of grip aperture in a double-step virtual grasping paradigm. Experimental Brain Research, v.125, n.1, Mar, p.61-6, 1999.

BOULINGUEZ, P.; NOUGIER, V. Control of goaldirected movements: the contribution of orienting of visual attention and motor preparation. Acta Psychologica (Amst), v.103, n.1-2, Nov, p.21-45, 1999.

BROWN, L. E.; HALPERT, B. A.; GOODALE, M. A. Peripheral vision for perception and action. Experimental Brain Research, v.165, n.1, Aug, p.97-106, 2005.

CALJOUW, S. R.; VAN DER KAMP, J.; SAVELSBERGH, G. J. Catching optical information for the regulation of timing. Experimental Brain Research, v.155, n.4, Apr, p.427-38, 2004.

CARNAHAN, H.; MARTENIUK, R. G. Hand, eye, and head coordination while pointing to perturbed targets. Journal of Motor Behavior, v.26, n.2, Jun, p.135-46, 1994.

DUBROWSKI, A.; LAM, J.; CARNAHAN, H. Target velocity effects on manual interception kinematics. Acta Psychologica (Amst), v.104, n.1, Mar, p.103-18, 2000.

ENGEL, K. C.; SOECHTING, J. F. Manual tracking in two dimensions. Journal of Neurophysiology, v.83, n.6, Jun, p.3483-96, 2000.

GEORGOPOULOS, A. P.; KALASKA, J. F.; MASSEY, J. T. Spatial trajectories and reaction times of aimed movements: effects of practice, uncertainty, and change in target location. Journal of Neurophysiology, v.46, n.4, Oct, p.725-43, 1981.

LAND, M. F.; MCLEOD, P. From eye movements to actions: how batsmen hit the ball. Nature Neuroscience, v.3, n.12, Dec, p.1340-5, 2000.

LE RUNIGO, C.; BENGUIGUI, N.; BARDY, B. G. Perception-action coupling and expertise in interceptive actions. Human Movement Science, v.24, n.3, Jun, p.429-45, 2005.

LEE, D. N.; GEORGOPOULOS, A. P.; CLARK, M. J.; CRAIG, C. M.; PORT, N. L. Guiding contact by coupling the taus of gaps. Experimental Brain Research, v.139, n.2, Jul, p.151-9, 2001.

LEE, D. N.; YOUNG, D. S.; REDDISH, P. E.; LOUGH, S.; CLAYTON, T. M. Visual timing in hitting an accelerating ball. Quarterly Journal of Experimental Psychology A, v.35, n.Pt 2, May, p.333-46, 1983.

MICHAELS, C. F.; ZEINSTRA, E. B.; OUDEJANS, R. R. Information and action in punching a falling ball. Quarterly Journal of Experimental Psychology A, v.54, n.1, Feb, p.69-93, 2001.

MILNER, A. D.; GOODALE, M. A. Two visual systems re-viewed. Neuropsychologia, v.46, n.3, p.774-85, 2008.

MONTAGNE, G.; LAURENT, M.; RIPOLL, H. Visual information pick-up in ball-catching. Human Movement Science, v.12, p.273-297, 1993.

MURPHY, K. J.; GOODALE, M. A. The effect of retinal eccentricity on implicit and explicit judgments of objects width. Society for Neuroscience Abstracts, v.20, p.1580, 1994.

PROTEAU, L.; BOIVIN, K.; LINOSSIER, S.; ABAHNINI, K. Exploring the limits of peripheral vision for the control of movement. Journal of Motor Behavior, v.32, n.3, Sep, p.277-86, 2000.

RODRIGUES, S. T.; VICKERS, J. N.; WILLIAMS, A. M. Head, eye and arm coordination in table tennis. Journal of Sports Sciences, v.20, n.3, Mar, p.187-200, 2002.

SAUNDERS, J. A.; KNILL, D. C. Visual feedback control of hand movements. Journal of Neuroscience, v.24, n.13, Mar 31, p.3223-34, 2004.

SAVELSBERGH, G. J.; WHITING, H. T.; BOOTSMA, R. J. Grasping tau. Journal of Experimental Psychology: Human Perception and Performance, v.17, n.2, May, p.315-22, 1991.

SMEETS, J. B.; BRENNER, E.; DE LUSSANET, $M$. H. Visuomotor delays when hitting running spiders. In: B. BRIL; A. LEDEBT; G. DITRICH; A. ROBY-BRAMI (Ed.). Advances in perceptionaction coupling. Paris: Éditions EDK, 1998. Visuomotor delays when hitting running spiders, p.36-40

TEIXEIRA, L. A.; CHUA, R.; NAGELKERKE, P.; FRANKS, I. M. Reprogramming of interceptive actions: time course of temporal corrections for unexpected target velocity change. Journal of Motor Behavior, v.38, n.6, Nov, p.467-77, 2006a.

TEIXEIRA, L. A.; CHUA, R.; NAGELKERKE, P.; FRANKS, I. M. Use of visual information in the correction of interceptive actions. Experimental Brain Research, v.175, n.4, Nov, p.758-63, 2006b. 
TEIXEIRA, L. A.; FRANZONI, M. M.; DA SILVA, J.

$B$. Are the elderly able to appropriately reprogram their actions? Motor Control, v.10, n.2, Apr, p.93108, 2006.

TEIXEIRA, L. A.; LIMA, E. D. S.; FRANZONI, M. $M$. The continuous nature of timing

reprogramming in an interceptive task. Journal of Sports Sciences, v.23, n.9, Sep, p.943-50, 2005.

TRESILIAN, J. R. Visually timed action: time-out for 'tau'? Trends Cognitive Sciences, v.3, n.8, Aug, p.301-310, 1999.

WILLIAMS, A. M.; DAVIDS, K.; WILLIAMS, J. G. Visual perception and action in sport. Londres: E \& FN Spon, 1999

WURTZ, R. H.; KANDEL, E. R. Perception of motion, depth, and form. In: E. R. KANDEL; J. H. SCHWARTZ; T. M. Jessell (Ed.). Principles of neural science. New York: McGraw-Hill, 2000. p.548-571

Nota: Trabalho derivado de dissertação de mestrado do primeiro autor. Agradecimentos: Os autores agradecem à FAPESP pela bolsa de estudos concedida ao segundo autor (\#2006/05336-3), ao CNPq pela bolsa concedida ao terceiro autor (\#308312/2006-6), a Marcos Duarte por disponibilizar o sistema optoeletrônico Qualysis, e a Natália Siqueira pelo trabalho de arte da Figura 1.

Endereço:

Luis Augusto Teixeira

Av. Prof. Mello Moraes, 65

Escola de Educação Física e Esporte, USP

São Paulo SP Brasil

05508-030

Telefone: (11) 3091.2129

e-mail: lateixeira@pq.cnpq.br

Recebido em: 11 de janeiro de 2010.

Aceito em: 31 de agosto de 2010.

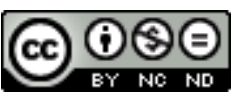

Motriz. Revista de Educação Física. UNESP, Rio Claro, SP, Brasil - elSSN: 1980-6574 - está licenciada sob Licenca Creative Commons 